\title{
Modulation of the sympathetic nervous system by renal denervation prevents reduction of aortic distensibility in atherosclerosis prone ApoE-deficient rats
}

Mathias Hohl ${ }^{1 *}$, Dominik Linz ${ }^{1}$, Peter Fries ${ }^{2}$, Andreas Müller², Jonas Stroeder ${ }^{2}$, Daniel Urban ${ }^{1}$, Thimoteus Speer ${ }^{3}$, Jürgen Geisel ${ }^{4}$, Björn Hummel ${ }^{5}$, Ulrich Laufs ${ }^{1}$, Stephan H. Schirmer ${ }^{1}$, Michael Böhm ${ }^{1}$ and Felix Mahfoud ${ }^{1}$

\begin{abstract}
Background: Apolipoprotein E-deficient $\left(\mathrm{ApoE}^{-/-}\right.$) rodents spontaneously develop severe hypercholesterolemia and increased aortic stiffness, both accepted risk factors for cardiovascular morbidity and mortality in humans. In patients with resistant hypertension renal denervation (RDN) may improve arterial stiffness, however the underlying mechanisms are incompletely understood. This study investigates the impact of RDN on aortic compliance in a novel atherosclerosis prone $\mathrm{ApoE}^{-/-}$-rat model.

Methods: Normotensive, 8 weeks old $\mathrm{ApoE}^{-/-}$and Sprague-Dawley (SD) rats were subjected to bilateral surgical RDN ( $n=6$ per group) or sham operation ( $n=5$ per group) and fed with normal chow for 8 weeks. Compliance of the ascending aorta was assessed by magnetic resonance imaging. Vasomotor function was measured by aortic ring tension recordings. Aortic collagen content was quantified histologically and plasma aldosterone levels were measured by enzyme-linked immunosorbent assay (ELISA).

Results: After 8 weeks, ApoE ${ }^{-1-}$-sham demonstrated a $58 \%$ decrease in aortic distensibility when compared with SD-sham ( $0.0051 \pm 0.0011$ vs. $0.0126 \pm 0.00231 / \mathrm{mmHg} ; \mathrm{P}=0.02)$. This was accompanied by an impaired endothelium-dependent relaxation of aortic rings and an increase in aortic medial fibrosis (17.87 \pm 1.4 vs. $12.27 \pm 1.1 \%$; $\mathrm{p}=0.006)$. In ApoE ${ }^{-/}$-rats, RDN prevented the reduction of aortic distensibility $(0.0128 \pm 0.002$ vs. $0.0051 \pm 0.0011$ $1 / \mathrm{mmHg} ; \mathrm{p}=0.01)$, attenuated endothelial dysfunction, and decreased aortic medial collagen content $(12.71 \pm 1.3$ vs. $17.87 \pm 1.4 \% ; p=0.01$ ) as well as plasma aldosterone levels (136.33 \pm 6.6 vs. $75.52 \pm 8.4 \mathrm{pg} / \mathrm{ml} ; p=0.0003$ ). Cardiac function and metabolic parameters such as hypercholesterolemia were not influenced by RDN.

Conclusion: $\mathrm{Apo}^{-/-}$-rats spontaneously develop impaired vascular compliance. RDN improves aortic distensibility and attenuated endothelial dysfunction in $\mathrm{Apo}^{-/-}$-rats. This was associated with a reduction in aortic fibrosis formation, and plasma aldosterone levels.
\end{abstract}

Keywords: ApoE-deficient rats, Hypercholesterolemia, Aortic distensibility, Renal sympathetic denervation

\section{Background}

The ability of the arterial system to adapt to changes in mechanical demands depends on its vascular compliance.

\footnotetext{
*Correspondence: Mathias.Hohl@uks.eu

1 Klinik für Innere Medizin III, Universität des Saarlandes,

66421 Homburg/Saar, Germany

Full list of author information is available at the end of the article
}

Compliance describes the amount of volume change of blood vessels following a given (blood) pressure change [1]. Hyperlipidemia can induce remodeling processes of the arterial wall, thereby impairing vascular compliance [2-4]. The underlying molecular mechanisms contributing to impaired vascular compliance are multifactorial and may involve increased sympathetic nerve activity leading to renin-angiotensin-aldosterone-system (RAAS) 
activation, enhanced vascular oxidative stress, and overexpression of inflammatory molecules [4-8].

Renal denervation (RDN) has been shown to reduce blood pressure (BP) in animal models and in certain patients with uncontrolled hypertension [9-12]. Recent studies have demonstrated that RDN may also exhibit additional systemic effects in parallel to BP lowering [13, 14], including improvement in arterial stiffness [15]. In obese, spontaneously hypertensive rats (SHR-ob), RDN inhibited progressive BP increase in SHR-ob and suppressed progression of kidney injury, cardiac fibrosis, and left ventricular dysfunction [16]. In goats with persistent atrial fibrillation (AF), RDN reduced atrial sympathetic nerve sprouting, structural alterations, and AF complexity [17]. In normotensive ApoE-deficient mice, fed with Western-type diet, RDN attenuated atherosclerotic lesion formation at the aortic root without affecting hyperlipidemia [18].

The effect of RDN on the development of aortic remodeling and impairment of vascular compliance under hyperlipidemic conditions yet at a preatherosclerotic stage has not yet been investigated systematically. To assess the role of the sympathetic nervous system and its modulation by RDN, we used a novel, normotensive ApoE-deficient rat model of hypercholesterolemia [19] with a hitherto uncharacterized cardiovascular phenotype.

\section{Methods}

All chemicals used were purchased from Carl Roth GmBH + Co.KG (Karlsruhe, Germany) unless mentioned otherwise.

\section{Animals}

The animal experiments were conducted in accordance with "The guide for the Care and Use of laboratory Animals" published by the US National Institutes of Health (NIH Publication No. 85-23, revised 1996) and approved by the local animal ethics committee (Landesamt für Verbraucherschutz, Amtstierärztlicher Dienst, Lebensmittelüberwachung Zentralstelle, Saarbrücken, Germany. Nr.: 38/2012). Male ApoE-deficient rats (\#TGRA3710HM4-1EA; ApoE ${ }^{-1-}, \mathrm{n}=11$ ), and their littermate controls Sprague-Dawley $(\mathrm{SD}, \mathrm{n}=11)$, were purchased from Sigma-Aldrich-SAGE Labs (Boyertown, USA) at an age of 7 weeks. The animals were housed individually in standard cages and received standard chow diet $\left(\right.$ Ssniff ${ }^{\circledR}$ standard diet \#V1536, Ssniff ${ }^{\circledR}$ Spezialitäten $\mathrm{GmbH}$, Soest, Germany) and tap water ad libitum. At an age of 8 weeks the animals were randomized to either RDN or sham operation and sacrificed at an age of 16 weeks. Blood pressure (BP) and heart rate (HR) were measured non-invasively by a computerized tail-cuff system (BP-2000 Visitech Systems, USA).

It is known from ApoE-deficient mice that usage of high-fat, high-cholesterol Western-type diet accelerated the progression of atherosclerosis $[18,20]$. In $\mathrm{ApoE}^{-/-}$. rats, we observed that administration of a Western-type diet containing $0.3 \%$ cholesterol resulted in increased mortality after $2-3$ weeks, most likely due to severe fatty degeneration of the liver (Additional file 1: Figure S1). Therefore, we resigned from feeding $\mathrm{ApoE}^{-1-}$-rats with Western-type diet and focused on preatherosclerotic aortic remodeling in hyperlipidemic $\mathrm{ApoE}^{-/-}$-rats at an age of 16 weeks fed with standard chow [19].

\section{Renal denervation and sham operation}

RDN in rats was performed as described previously [16]. Six $\mathrm{ApoE}^{-/-}$and six SD rats (ApoE ${ }^{-/-}-\mathrm{RDN}$ and SD-RDN, respectively) underwent RDN at the age of 8 weeks. In brief, rats were anesthetized with 1.5-2.5\% isoflurane (AbbVie \#B506, Ludwigshafen, Germany) and both kidneys were surgically denervated by cutting all visible nerves in the area of the renal hilus and by stripping approximately $2-4 \mathrm{~mm}$ of the adventitia from the renal artery and moistening with a $20 \%$ phenol/ethanol solution. Another five $\mathrm{ApoE}^{-/-}$and five SD rats were submitted to a surgical sham procedure with kidney exposition but without RDN, serving as sham controls $\left(\mathrm{ApoE}^{-1-}\right.$-sham and $\mathrm{SD}$-sham, respectively). At an age of 16 weeks, rats were sacrificed and reductions in renal tissue norepinephrine (NE) concentrations were determined by high-performance liquid chromatography to assess the effectiveness of RDN.

\section{Magnetic resonance imaging}

All MRI experiments were performed using a 9.4 Tesla horizontal bore animal scanner (Bruker BioSpin 94/20, Ettlingen, Germany) with a $2 \times 2$ phased-array surface coil. All animals were subjected to general anesthesia using a mixture of isoflurane and oxygene ( $2 \% / 98 \%)$ applied by a dedicated nose mask at a flow rate of $1.5 \mathrm{l} /$ min. A retrospectively self-gated black blood sequence (IntraGate FLASH, TR $8.9 \mathrm{~ms}$, TE $2.1 \mathrm{~ms}$, flip angle $10^{\circ}$, field of view $4.5 \times 4.5 \mathrm{~cm}$, matrix $384 \times 384$, slice thickness $1 \mathrm{~mm}$, pixel size $117 \times 117 \mu^{2}$ ) was acquired perpendicular to the ascending aorta at the level of the right pulmonary artery. 25 cine frames were reconstructed from the acquired raw data in order to display one cardiac cycle. In addition, retrospectively self-gated bright blood cine sequence were acquired in short axis orientation covering the left ventricle from base to apex with reconstruction of 20 cine frames for every dedicated slice position (IntraGate FLASH, TR $5.6 \mathrm{~ms}$, TE $1.5 \mathrm{~ms}$, flip angle $12^{\circ}$, field of view $4.0 \times 4.0 \mathrm{~cm}$, matrix $256 \times 256$, 
slice thickness $1 \mathrm{~mm}$, pixel size $\left.156 \times 156 \mu \mathrm{m}^{2}\right)$. For further quantitative analyses, the image data were transferred to an external workstation and evaluated using image evaluation software (Osiri ${ }^{\circledR}$, Pixmeo, Bernex, Switzerland).

For the assessment of aortic distensibility the cross sectional vessel area of the ascending aorta was measured by manual contouring at the blood/vessel wall interface at end-diastole (ED) and end-systole (ES).

Aortic distensibility (AD) was calculated with

$$
\begin{aligned}
\mathrm{AD}= & (\text { Area }(\mathrm{ES})-\text { Area }(\mathrm{ED}) / \\
& (\text { Area }(\mathrm{ED}) \times \text { pulse pressure }(\mathrm{PP})),
\end{aligned}
$$

Left ventricular end diastolic volume (LVEDV) and the left ventricular end systolic volume (LVESV) were evaluated by manual contouring of the blood/subendocardial myocardium.

Stroke volume (SV) and ejection fraction (EF) were calculated with:

$$
\begin{aligned}
& \mathrm{SV}=\mathrm{LVEDV}-\mathrm{LVESV} \\
& \mathrm{EF}=\mathrm{SV} / \mathrm{LVEDV}
\end{aligned}
$$

\section{Histology}

\section{Aortic medial collagen content}

Thoracic aorta was sectioned $(10 \mu \mathrm{m})$ on a Leica cryostat at $-25{ }^{\circ} \mathrm{C}$ (Kryostat Leica CM 1900-V5.0, Leica Microsystems, Nussloch, Germany). Sections were stained with Picro-Sirius Red (Morphisto ${ }^{\circledR}$, Frankfurt, Germany) to visualize collagen fibers. The percentage of the media consisting of interstitial collagen was calculated as the ratio of Picro-Sirius-Red positively stained area over total aortic medial-tissue area. For the analysis Nicon Instruments Software (NIS)-Elements (BR 3.2, Nikon instruments, USA) was used.

\section{Ficoll-isolation of mononuclear cells}

Peripheral blood mononuclear cells (MNCs) were isolated by the use of a Ficoll density gradient within $30 \mathrm{~min}$ after collecting peripheral arterial blood from all animals. Whole citrate blood was mixed 1:1 (v/v) with a solution of phosphate-buffered saline (PBS) and layered onto 0.25 Vol. of Ficoll (Biochrom GmbH, Berlin, Germany). After centrifugation at $800 \mathrm{~g}$ for $20 \mathrm{~min}$ at room temperature, the layer of mononuclear cells was collected and stored immediately for RNA isolation.

\section{Gene expression}

Reverse Transcription: RNA was prepared from rat aortic-tissue and mononuclear cells using peqGold TriFast (PeqLab, Erlangen, Germany) extraction reagent per manufacturer's protocol. For cDNA preparation $2 \mu \mathrm{g}$ of RNA were digested with DNAse (Peqlab) than reversely transcribed using the HighCap cDNA RT Kit (Applied Biosystems, Darmstadt, Germany) according to the manufacturer's protocol. TaqMan PCR was conducted in a StepOne plus thermocycler (Applied Biosystems) using TaqMan GenEx Mastermix (Applied Biosystems, \#4369016). Signals were normalized to corresponding glyceraldehyde-3-phosphate dehydrogenase (GAPDH) controls. No template controls were used to monitor for contaminating amplifications. The $\Delta \mathrm{Ct}$ was used for statistical analysis and $2^{-\Delta \Delta C t}$ for data presentation. Probes used to amplify the transcripts were as follows (Applied Biosystems): TNFa (Rn01525859_g1); IL1 $\beta$ (Rn00580432_m1); IL6 (Rn01410330_m1); GAPDH (Rn99999916_s1), ICAM1 (Rn00564227_m1), VCAM1 (Rn00563627_m1); eNOS (Rn02132634_s1).

\section{Enzyme linked immunosorbent assays (ELISA)}

Plasma concentrations of aldosterone, angiotensin II, IL6 and IL1 $\beta$ were determined by ELISA, using Enzo Aldosterone EIA Kit (ADI-900-173; Lausen, Switzerland), Enzo Angiotensin II ELISA Kit (ADI-900-204; Lausen, Switzerland), Rat IL6 ELISA Kit (R\&D systems, Abington, UK) and Cloud IL1 $\beta$ Immunoassay (Cloud-Clone Corp. Houston, TX, USA) following the manufacturer's protocol. For analysis of renal renin and angiotensin II tissue concentrations, $10 \mathrm{mg}$ kidney of each rat was homogenized in $600 \mu \mathrm{l}$ PBS, containing $1 \%$ Triton- $X$ and complete protease inhibitors (Roche Diagnostics GmbH, Mannheim, Germany), followed by centrifugation at $18000 \mathrm{~g}$ for $5 \mathrm{~min}$. Angiotensin II and renin levels were measured from the supernatant by ELISA using Elabscience ELISA Kit (WuHan, Peoples Republic of China) following the manufacturer's protocol.

\section{Determination of renal norepinephrine content}

Kidney tissue from rat was minced thoroughly in TRISEDTA buffer (in mmol/l: 5.0 Tris(hydroxymethyl)aminomethane, 2.0 Na-EDTA, pH 7.4). After centrifugation, supernatant was used to analyze NE content by high pressure liquid chromatography (HPLC) using Chromosystems catecholamine Kit for HPLC (Chromsystems Instruments \& Chemicals GmbH, Munich, Germany) following the manufacturer's protocol.

\section{Aortic ring preparation and tension recording}

Aortic ring tension was measured as described previously [20]. After excision, the thoracic aorta was immersed in Tyrode's solution containing (in mmol/l) $118.0 \mathrm{NaCl}, 2.5$ $\mathrm{CaCl}_{2}, 4.73 \mathrm{KCl}, 1.2 \mathrm{MgCl}_{2}, 1.2 \mathrm{KH}_{2} \mathrm{PO}_{4}, 25.0 \mathrm{NaHCO}_{3}$, $0.026 \mathrm{Na}$-EDTA, and $5.5 \mathrm{D}-(+)$-glucose, $\mathrm{pH}$ 7.4. Adventitial tissue was carefully removed. Three-millimeter aortic rings $(\mathrm{n}=4$ rings per animal with $\mathrm{n}=5$ animals per group) were mounted in organ bath chambers filled with the Tyrode's solution $\left(37^{\circ} \mathrm{C}\right.$; aerated with $95 \% \mathrm{O}_{2}$ 
and $\left.5 \% \mathrm{CO}_{2}\right)$ and were attached to a force transducer recording isometric tension (Model 750TOBS, Danish Myo Technology (DMT), Aarhus, Denmark). Aortic rings were stretched to a resting tension of $10 \mathrm{mN}$, which was maintained throughout the experiment. Pharmacologically induced contraction of aortic rings was performed with phenylephrine $(5 \mu \mathrm{mol} / \mathrm{l}$; Sigma-Aldrich, Steinheim, Germany). After a plateau was reached, increasing concentrations of carbachol (carbamylcholine chloride; $1 \mathrm{nmol} / \mathrm{l}-100 \mu \mathrm{mol} / \mathrm{l}$; Sigma-Aldrich) were added to obtain cumulative concentration-response curves to study endothelium-dependent relaxation. The relaxing effect of carbachol was abolished by adding $N$-nitro-L-arginine methyl ester $(1 \mu \mathrm{mol} / \mathrm{l}$; Sigma-Aldrich). To study endothelium-independent relaxation after a washout period, increasing concentrations of glyceryl trinitrate (100 nmol/l-10 $\mu \mathrm{mol} / \mathrm{l}$; Sigma-Aldrich) were added after a single dose of phenylephrine $(5 \mu \mathrm{mol} / \mathrm{l})$.

Aortic rings without any response to relaxation with carbachol or glyceryl trinitrate $(<10 \%$ relaxation) were excluded from statistical analysis. Relaxation response was expressed as percentage of phenylephrine-induced pre-contraction. The concentration of drugs used to achieve $50 \%$ of the maximal response $\left(\operatorname{logEC_{50}}\right)$ was determined by using nonlinear regression analysis (GraphPad Prism 6, San Diego, CA, USA).

\section{Statistics}

Data are presented as mean \pm SEM and differences were tested for significance using an unpaired Student's $t$ test, one-way-ANOVA with Tukey's multiple comparisons test or two-way-ANOVA with Bonferroni multiple comparisons test when appropriate. A p value of less than 0.05 was considered statistically significant. Statistical analysis was performed using GraphPad Prism 6.

\section{Results}

Renal norepinephrine content, cardiac function, and metabolic parameters

Eight weeks after RDN-procedure, renal NE concentrations were significantly lower in SD-RDN and $\mathrm{ApoE}^{-1-}$ $\mathrm{RDN}$, when compared to their respective sham-operated controls (Fig. 1), confirming successful renal nerve ablation. Table 1 depicts parameters of cardiac function and serum chemistry eight weeks after RDN- or sham intervention. Body weight, heart weight, systolic and diastolic blood pressure, heart rate, stroke volume, ejection fraction, left ventricular systolic, and diastolic volume did not differ between $\mathrm{ApoE}^{-1-}$-sham and SD-sham, demonstrating that at an age of 16 weeks $\mathrm{ApoE}^{-1-}$-rats remain normotensive. Blood pressure did not differ between $\mathrm{ApoE}^{-1-}$ and SD rats at the age of 8 weeks. Total cholesterol, low-density lipoprotein (LDL), and triglycerides

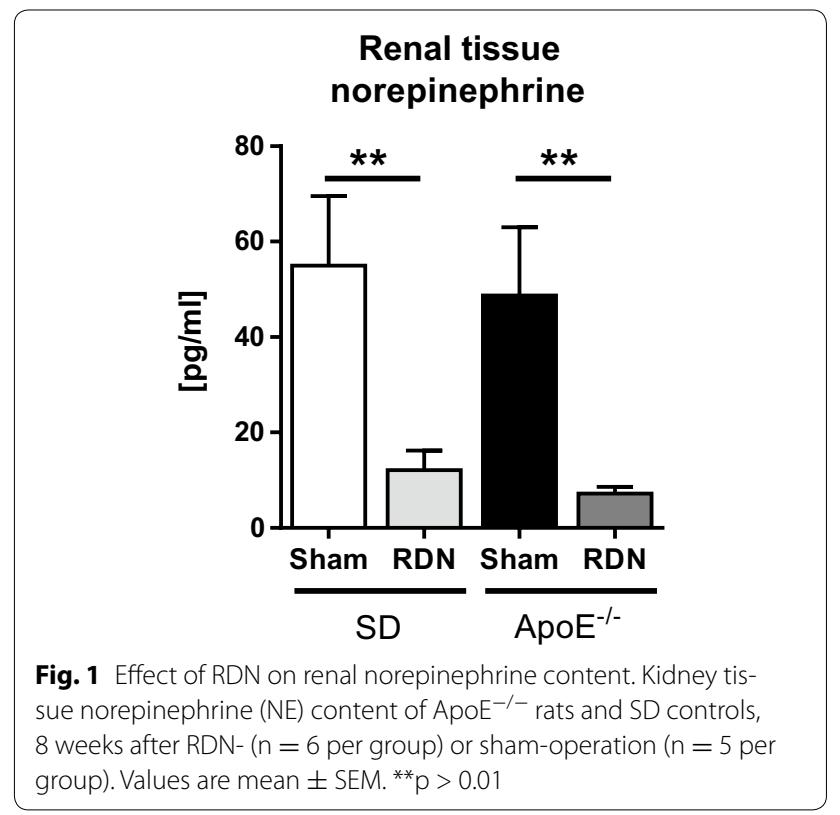

were significantly higher in $\mathrm{ApoE}^{-/-}$-rats, while highdensity lipoprotein (HDL) was significantly lower. RDN had no influence on myocardial function or metabolic parameters.

\section{RDN improved aortic distensibility and attenuated progression of endothelial-dependent dysfunction} At an age of 16 weeks, $\mathrm{ApoE}^{-1-}$-sham rats exhibited a significantly reduced aortic distensibility compared with age-matched SD-rats $(0.0051 \pm 0.0011$ vs. $0.0126 \pm 0.00231 / \mathrm{mmHg} ; \mathrm{p}=0.02$ ), as measured by MRI with high-resolution cine sequences acquired at 9.4 Tesla. ApoE ${ }^{-1-}$-rats also demonstrated an agedependent reduction of aortic distensibility, comparing baseline values at an age of 8 weeks $(0.0138 \pm 0.00391 /$ $\mathrm{mmHg}$ ) and final measurements at an age of 16 weeks $(0.0051 \pm 0.00111 / \mathrm{mmHg} ; \mathrm{p}=0.07)$. Lack of statistical significance might by due to the limited number of animals.

In $\mathrm{ApoE}^{-1-}$-RDN rats, reduction of aortic distensibility was prevented $(0.0128 \pm 0.002(1 / \mathrm{mmHg} ; \mathrm{p}=0.01$ vs. ApoE-sham) and equals values of SD-sham rats (Fig. 2a, b). Endothelial relaxation of aortic rings was tested in organ bath experiments at an age of 16 weeks. Efficacy of endothelium-dependent vascular response to increasing concentrations of carbachol was significantly impaired in $\mathrm{ApoE}^{-1-}$-sham compared with SD-sham. In $\mathrm{ApoE}^{-1-}$ receiving $\mathrm{RDN}$, vasorelaxation improved when compared with $\mathrm{ApoE}^{-1-}$-sham animals (Fig. 3a). Calculation of the maximal response $\left(E_{\max }\right)$ and half-maximal dose $\left(\log \mathrm{EC}_{50}\right)$ to Carbachol also demonstrated a reduced 
Table 1 Body weight, blood pressure and metabolic parameters 8 weeks after RDN

\begin{tabular}{|c|c|c|c|c|}
\hline & SD-sham $n=5$ & SD-RDN $n=6$ & $\mathrm{ApoE}^{-/-}-\operatorname{sham} \mathrm{n}=5$ & $\mathrm{ApoE}^{-l-}-\mathrm{RDN} \mathrm{n}=6$ \\
\hline Body weight (g) & $456.2 \pm 25.64$ & $474.14 \pm 11.26$ & $446.75 \pm 3.68$ & $456.2 \pm 10$ \\
\hline Heart weigth (g) & $1.41 \pm 0.04$ & $1.45 \pm 0.05$ & $1.51 \pm 0.06$ & $1.45 \pm 0.04$ \\
\hline Heart rate (bpm) & $360 \pm 9$ & $370 \pm 10$ & $341 \pm 13$ & $359 \pm 7$ \\
\hline \multicolumn{5}{|l|}{ Blood pressure $(\mathrm{mmHg})$} \\
\hline Systolic & $144 \pm 5$ & $137 \pm 4$ & $148 \pm 12$ & $136 \pm 7$ \\
\hline Diastolic & $116 \pm 4$ & $109 \pm 10$ & $121 \pm 11$ & $99 \pm 8$ \\
\hline Stroke volume $(\mu \mathrm{l})$ & $210 \pm 10$ & $219 \pm 5$ & $203 \pm 10$ & $224 \pm 9$ \\
\hline Ejection fraction (\%) & $61 \pm 3$ & $61 \pm 1$ & $59 \pm 2$ & $60 \pm 2$ \\
\hline $\operatorname{LVEDV~}(\mu \mathrm{l})$ & $342 \pm 11$ & $357 \pm 11$ & $344 \pm 8$ & $372 \pm 13$ \\
\hline LVESV $(\mu l)$ & $131 \pm 12$ & $137 \pm 8$ & $140 \pm 11$ & $148 \pm 10$ \\
\hline \multicolumn{5}{|l|}{ Serum chemistry } \\
\hline GPT (U/I) & $79.64 \pm 7.53$ & $70.5 \pm 5.91^{*}$ & $109.66 \pm 14.96$ & $104.43 \pm 10.26$ \\
\hline GOT (U/I) & $178.36 \pm 24.74$ & $169.64 \pm 13.83$ & $222.22 \pm 33.31$ & $217.28 \pm 32.1$ \\
\hline Creatinine (mg/dl) & $0.306 \pm 0.02$ & $0.33 \pm 0.01$ & $0.33 \pm 0.02$ & $0.29 \pm 0.03$ \\
\hline Glucose (mg/dl) & $235.2 \pm 8.24$ & $268.71 \pm 17.18$ & $328.25 \pm 28.51$ & $289.33 \pm 25.65$ \\
\hline Cholesterol (mg/dl) & $48.2 \pm 3.28^{\S}$ & $45.71 \pm 3.21^{\#}$ & $131.5 \pm 17.54$ & $152.5 \pm 13.63$ \\
\hline Triglycerides (mg/dl) & $30.6 \pm 7.04^{\S}$ & $30.57 \pm 8.05^{\#}$ & $152.2 \pm 27.41$ & $152.5 \pm 18.44$ \\
\hline $\mathrm{LDL}(\mathrm{mg} / \mathrm{dl})$ & $10.96 \pm 1.15^{\S}$ & $10.5 \pm 0.94^{\#}$ & $52.3 \pm 6.62$ & $65.28 \pm 9.61$ \\
\hline $\mathrm{HDL}(\mathrm{mg} / \mathrm{dl})$ & $37.58 \pm 1.36^{\S}$ & $34.93 \pm 3.36^{\#}$ & $11.7 \pm 1.67$ & $15.78 \pm 1.81$ \\
\hline
\end{tabular}

Values are mean \pm SEM

LVEDV left ventricular end-diastolic volume, LVESV left ventricular end-systolic volume, GPT glutamat-pyruvat-transaminase, GOT glutamat-oxalacetat-transaminase, $H D L$ high-density lipoprotein, $L D L$ low-density lipoprotein

* $\mathrm{p}<0.05$ SD-RDN vs. ApoE ${ }^{-1-}-\mathrm{RDN}$

$\S p<0.01$ SD-sham vs. ApoE ${ }^{-1-}$-sham

\# $\mathrm{p}<0.01 \mathrm{SD}-\mathrm{RDN}$ vs. ApoE ${ }^{-\backslash-}$-RDN (one-way-Anova followed by Tukey's multiple comparisons test)

response of the endothelium in $\mathrm{ApoE}^{-/-}$-sham compared to SD-sham. However, RDN-treatment of $\mathrm{ApoE}^{-1-}$ failed to reach significant improvement of endothelial relaxation using linear regression calculation (Table 2). Endothelium-independent relaxation induced by glyceryl trinitrate did not significantly differ between the groups (Fig. 3b). $E_{\max }$ and $\log \mathrm{EC}_{50}$ of glyceryl trinitrate did also not differ among the groups (Table 2).

Additionally, we analyzed mRNA expression pattern of endothelial nitric oxide synthase (eNOS) in aortic tissue of $\mathrm{ApoE}^{-1-}$ and SD rats. Levels of mRNA were not differentially regulated between the groups and RDN had no effect on eNOS gene expression (Additional file 1: Table S1).

\section{Effect of RDN on RAAS activation and inflammatory response}

Plasma aldosterone concentration was significantly increased in $\mathrm{ApoE}^{-1-}$-sham compared with SD-sham $(136.33 \pm 6.6$ vs. $88.83 \pm 15.4 \mathrm{pg} / \mathrm{ml} ; \mathrm{p}=0.02)$. Enhanced aldosterone release was inhibited following RDN in ApoE $^{-1-}(75.52 \pm 8.4 \mathrm{pg} / \mathrm{ml} ; \mathrm{p}=0.0003$ vs. ApoE-sham $)$, but not in SD-RDN $(99.17 \pm 12.2 \mathrm{pg} / \mathrm{ml} ; \mathrm{p}=0.60 \mathrm{vs}$. SDsham) (Fig. 4a). AngII plasma level was not changed in ApoE $^{-1-}$-sham compared with SD-sham $(25.26 \pm 6.2$ vs. $14.92 \pm 2.3 \mathrm{pg} / \mathrm{ml} ; \mathrm{p}=0.15)$ and $\mathrm{RDN}$-treatment did not significantly reduce AngII plasma level neither in ApoE $^{-l-}$-RDN $(17.51 \pm 4.1 \mathrm{pg} / \mathrm{ml} ; \mathrm{p}=0.07$ vs. ApoEsham) nor in SD-RDN $(9.82 \pm 1.4 \mathrm{pg} / \mathrm{ml} ; \mathrm{p}=0.08$ vs. SD-sham). Renal tissue concentration of renin and AngII did not differ between sham-operated rats, however RDN resulted in a significant reduction of renin and AngII-tissue level in $\mathrm{ApoE}^{-/-}$-RDN and SD-RDN (Fig. 4b).

In circulating mononuclear cells, gene expression of TNF $\alpha$ and IL6 was significantly increased in $\mathrm{ApoE}^{-1-}$-sham compared with SD-sham, while IL1 $\beta$ was unchanged. RDN significantly inhibited transcriptional enhancement of TNF $\alpha$ and IL6 in $\mathrm{ApoE}^{-1-}$, but had no effect on IL1 $\beta$ mRNA-levels (Fig. 4c). Plasma levels of IL6 and IL1 $\beta$ were significantly higher in $\mathrm{ApoE}^{-1-}$ sham compared to SD-sham (IL6: $157.06 \pm 6.54$ vs. $123.78 \pm 4.39 \mathrm{pg} / \mathrm{ml}, \mathrm{p}<0.01$; IL1 $13: 177.41 \pm 8.77$ vs. $65.75 \pm 15.32 \mathrm{pg} / \mathrm{ml}, \mathrm{p}<0.01)$ demonstrating increased inflammation in $\mathrm{ApoE}^{-/-}$rats. Modulation of the sympathetic nervous system by RDN had no effect on IL6 or IL1 $\beta$ plasma concentration, neither in SD-RDN nor in $\mathrm{ApoE}^{-1-}$-RDN (Additional file 1: Table S1).

In aortic tissue of $\mathrm{ApoE}^{-1-}$-sham, IL1 $\beta$ mRNA-levels were elevated in comparison to SD-sham (relative gene 

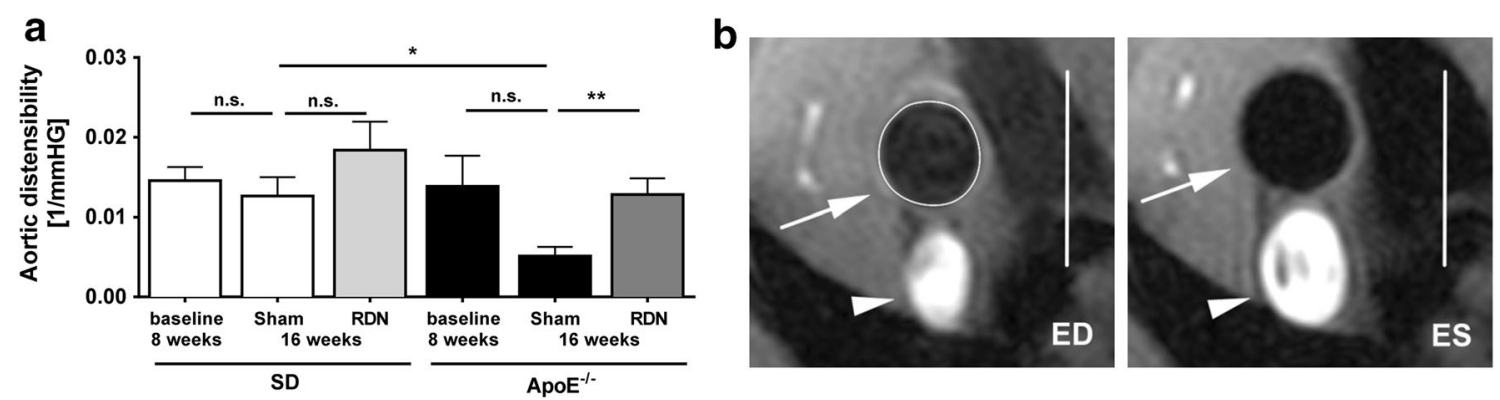

Fig. 2 Effect of RDN on aortic distensibility and vasorelaxation. a Quantification of aortic distensibility at baseline ( $n=6$ per group), and 8 weeks after RDN procedure ( $n=6$ per group) or sham operation ( $n=5$ per group). Data are based on in vivo measurements of cross section vessel areas. b Representative example of high-resolution black blood cine MRI perpendicular to the ascending aorta. The bar on the right side indicates $5 \mathrm{~mm}$. The circle depicts the cross sectional vessel area of the ascending aorta (arrow). The arrowhead points to the superior caval vein. ED end diastole, ES end systole. Values are mean \pm SEM. SD baseline vs. SD-sham, $p=0.51$ (n.s.); SD-sham vs. SD-RDN, $p=0.23$ (n.s.); ApoE ${ }^{-/-}$baseline vs. ApoE $^{-/-}$ sham, $p=0.07$ (n.s.); ${ }^{*}<0.05,{ }^{* *} p<0.01$

\section{a}

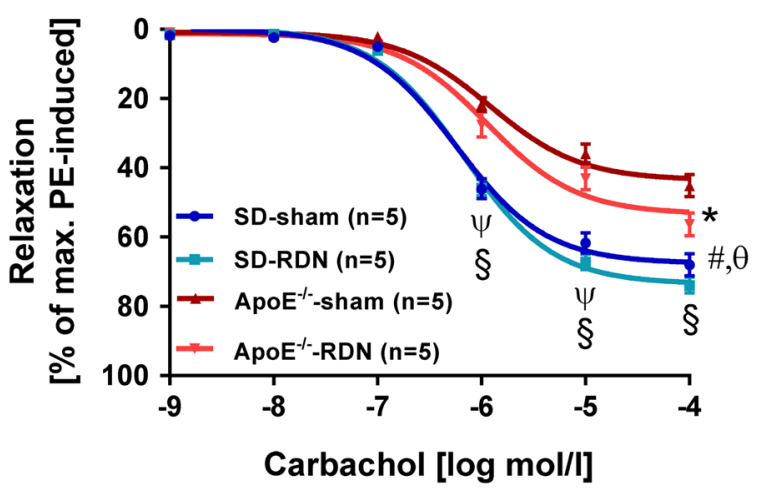

\section{b Endothelium-independent relaxation}

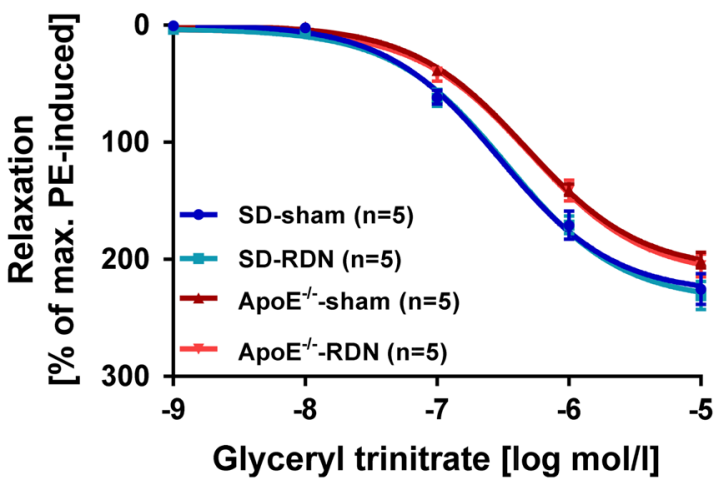

Fig. 3 Effect of RDN on endothelial function. a Endotheliumdependent vasorelaxation of aortic rings in response to carbachol and $\mathbf{b}$ endothelium-independent relaxation in response to glyceryl trinitrate in 16 weeks old SD-sham, SD-RDN, $\mathrm{ApoE}^{-/-}$-sham and ApoE ${ }^{-/-}$-RDN rats. Values are mean \pm SEM. ${ }^{8} \mathrm{p}<0.001$ SD-RDN vs. ApoE ${ }^{-\backslash}-$ RDN and $\mathrm{ApoE}^{-\backslash-}$-sham; ${ }^{\psi} \mathrm{p}<0.001$ SD-sham vs. ApoE ${ }^{-\backslash-}$ RDN;\#p $<0.01$ SD-sham vs. ApoE ${ }^{-\backslash}-$ RDN; ${ }^{\theta} p<0.001$ SD-sham vs. ApoE ${ }^{-\backslash-}$-sham; ${ }^{*} \mathrm{p}<0.01 \mathrm{ApoE}^{-\backslash-}$-RDN vs. ApoE ${ }^{-\backslash-}$-sham (two-wayAnova followed by Bonferroni multiple comparison test)
Table 2 Maximal relaxation $\left(E_{\max }\right)$ and half-maximal dose $\left(\log \mathrm{EC}_{50}\right)$ in response to carbachol and glyceryl trinitrate in 16 weeks old rats

\begin{tabular}{lllll}
\hline & SD-sham & SD-RDN & $\begin{array}{l}\text { ApoE }^{-1-} \\
\text { sham }\end{array}$ & $\begin{array}{l}\text { ApoE }^{-1-} \\
\text { RDN }\end{array}$ \\
\hline $\begin{array}{l}\text { Number of rats } \\
\text { Response to carbachol }\end{array}$ & 5 & 5 & 5 \\
$E_{\text {max }}(\%)$ & $67 \pm 6.0$ & $73 \pm 3.3$ & $43 \pm 4.8^{\S}$ & $53 \pm 4.9$ \\
$\log C_{50}(\log M)$ & $-6.22 \pm 0.06$ & $-6.17 \pm 0.03$ & $-5.81 \pm 0.15$ & $-5.88 \pm 0.12$ \\
Response to glyceryl trinitrate & & & \\
$E_{\text {max }}(\%)$ & $232 \pm 24$ & $237 \pm 6$ & $209 \pm 13$ & $217 \pm 16$ \\
$\log C_{50}(\log M)$ & $-6.5 \pm 0.10$ & $-6.47 \pm 0.08$ & $-6.32 \pm 0.06$ & $-6.29 \pm 0.10$
\end{tabular}

Values are mean \pm SEM. $p$ values were calculated using one-way-Anova followed by Tukey's multiple comparisons test

$\S \mathrm{p}=0.01 \mathrm{SD}$-sham vs. ApoE ${ }^{-\backslash-}$-sham

expression/GAPDH: $2.55 \pm 0.3$ vs. $1.15 \pm 0.2 ; \mathrm{p}=0.02$ ), while transcription of TNF and pro-atherosclerotic intercellular adhesion molecule-1 (ICAM-1) and vascular cell adhesion molecule 1 (VCAM-1) were unchanged. RDN could neither attenuate inflammatory nor pro-atherosclerotic gene expression (Additional file 1: Table S1).

\section{Morphological analysis of the thoracic aortic wall}

In 16 weeks old $\mathrm{ApoE}^{-/-}$and $\mathrm{SD}$ rats, microscopic analysis of the aorta showed no detectable atherosclerotic plaque formation in the open luminal surface after staining with Oil-red $\mathrm{O}$, and no visible fragmentation of elastin fibers after staining with Elastin van Gieson (Additional file 1: Figure S2). However, Sirius-red staining demonstrated an increased amount of interstitial fibrosis in the aortic media of $\mathrm{ApoE}^{-1-}$-sham compared with SD-sham (Fig. 5a). Collagen content was significantly lower in $\mathrm{ApoE}^{-1-}$-RDN compared with $\mathrm{ApoE}^{-/-}$sham (Fig. 5b). Wall thickness of the aortic media, 


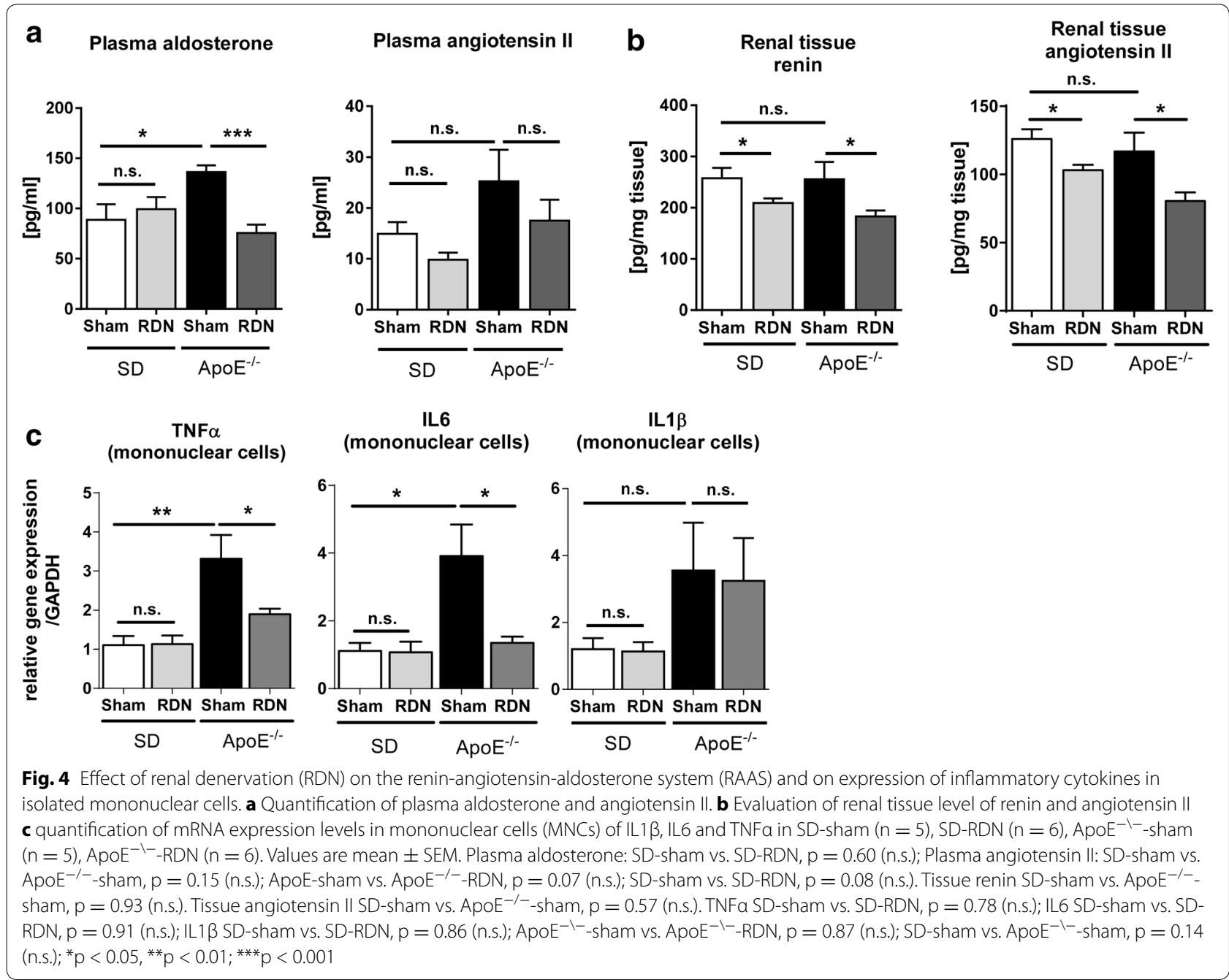

assessed by hematoxylin-eosin staining, was unchanged in $\mathrm{ApoE}^{-/-}$-sham compared to SD-sham $(124 \pm 9.8$ vs. $105 \pm 1.7 \mu \mathrm{m} ; \mathrm{p}=0.13$ ), and was not influenced by RDN (SD-RDN: $103 \pm 5.8 \mu \mathrm{m}$; ApoE $^{-l-}-\mathrm{RDN}: 113 \pm 7.3 \mu \mathrm{m}$; Additional file 1: Figure S2).

\section{Discussion}

The sympathetic nervous system (SNS) is involved in the induction and progression of multiple cardiovascular pathologies such as vascular damage $[7,8]$, atrial fibrillation [14], increased heart rate [21], and hypertension [22]. Modulation of the SNS by RDN has been shown to reduce BP in certain patients with uncontrolled hypertension [9-12]. Recent studies have demonstrated that RDN may also exhibit additional systemic effects, occurring either in combination with blood-pressure lowering [13, 14], such as improvement of arterial stiffness in patients with hypertension [15], or being partly independent of blood pressure changes [16-18]. The underlying mechanisms of these beneficial effects on vascular compliance are currently incompletely understood. The present study therefore aimed to further elucidate on the role of the SNS and its modulation by RDN on aortic remodeling and compliance in a atherosclerosis prone animal model of hyperlipidemia. We used a novel normotensive, hypercholesterolemic ApoE-deficient rat model [19], and demonstrated that these rats exhibit severely decreased aortic distensibility and an impaired vasorelaxation, already at a stage before atherosclerotic lesions could be detected histologically in the aortic sinus or the thoracic aorta. Our main findings are, that sympathetic modulation by surgical bilateral RDN improved aortic compliance and attenuated endothelial dysfunction. These improvements were associated with a decrease in circulating aldosterone levels, reduced aortic medial fibrosis formation, as well as a lower inflammatory cytokine expression in circulating mononuclear cells (MNCs). 

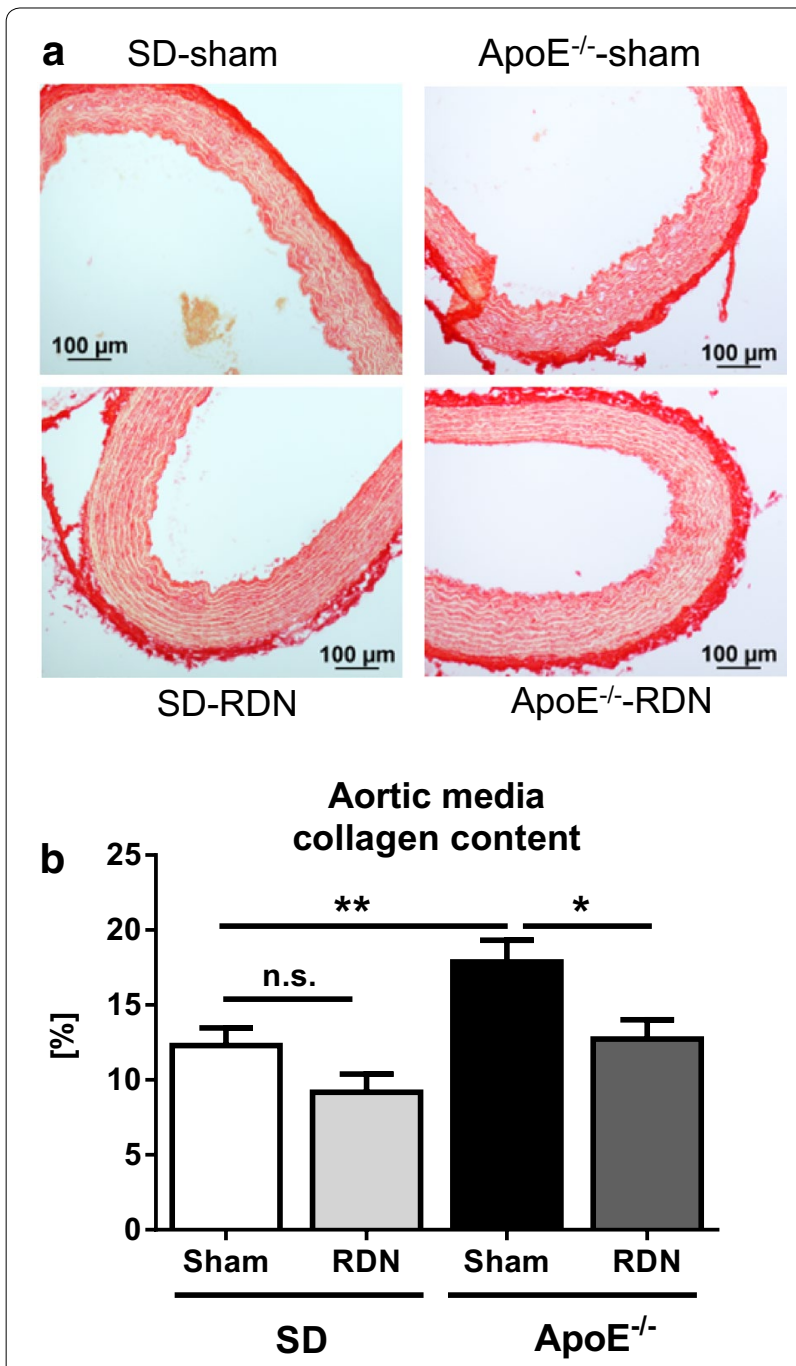

Fig. 5 Effect of RDN on aortic medial fibrosis content. a Representative Sirius-red staining for determination of interstitial collagen content of the aortic media. b Quantification of collagen content

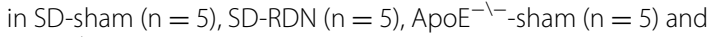
ApoE ${ }^{-\backslash-}-R D N(n=5)$. Values are mean \pm SEM. SD-sham vs. SD-RDN, $p=0.07$ (n.s.); ${ }^{*} p<0.05 ;{ }^{* *} p<0.01$

The renal SNS controls the renin secretion from the kidneys, thereby regulating the activity of the reninangiotensin-aldosterone system (RAAS). Renin release stimulates the generation of angiotensin II (AngII), and the release of aldosterone from the adrenal cortex [23]. AngII has been linked to structural alterations of the aortic wall, characterized by elastin deposition, increase in medial wall thickness, and vascular fibrosis formation [24], accompanied by an enhanced expression of inflammatory cytokines, which in the long-term result in vascular stiffness $[4,6,24,25]$. Accordingly, blockade of AngII decreases medial wall thickness and vascular fibrosis [20, 24, 25]. Selective aldosterone receptor blockade by eplerenone has been shown to reduce atherosclerosis and improve vasorelaxation in non-human primates fed with a high-cholesterol diet [26]. In $\mathrm{ApoE}^{-/-}$mice, administration of eplerenone prevented atherosclerosis progression without affecting serum cholesterol or triglyceride levels [27]. This is in line with recent findings demonstrating that RDN decreases serum aldosterone and atherosclerotic lesion formation in the aortic roots of $\mathrm{ApoE}^{-1-}$-mice, independently of changes in BP [18]. Consistently with these reports, in the novel $\mathrm{ApoE}^{-/-}$rat model used herein, RDN reduced plasma aldosterone levels and attenuated aortic medial fibrosis formation, while hyperlipidemia and cardiac parameters were not affected. Although RDN did not significantly influence blood pressure herein, it is conceivable that even a moderate decrease of BP may contribute to the herein observed improvements.

The aortic distensibility is codetermined by structural components of the arterial wall, i.e. extracellular matrix proteins $[5,28]$. In the present study, $\mathrm{ApoE}^{-/-}$rats demonstrated decreased aortic distensibility, associated with an increased aortic collagen content. This structural alteration was inhibited by RDN, preserving aortic distensibility, and emphasizes the influence of the SNS on aortic wall components. Vascular compliance, however, does not only depend on its wall composition, but also on endothelial function. Herein, modulation of the SNS by RDN positively influences endothelium-dependent relaxation which also might beneficially affect endothelial function. Endothelial function also highly depends on nitric oxide (NO)-bioavailability, regulated by the endothelial NO synthase (eNOS) [29, 30]. In ApoE-mice fed with high-fat diet down-regulation of eNOS was associated with impaired vascular function [20]. However, in our ApoE-deficient rat model, eNOS mRNA expression was not altered. It may also be conceivable, that local AngII mediates uncoupling of eNOS, thus decreasing total NO content and causing endothelial dysfunction [31]. The mechanisms regulation endothelial function are manifold and need to be further elucidated for this novel rat model.

Inflammation was also shown to initiate structural changes in the composition of the arterial wall, contributing to arterial stiffening [6, 32]. Several cytokines (e.g. IL1 $\beta$, IL6, and TNF $\alpha$ ) are known to stimulate the expression of ICAM-1 and VCAM-1, two important molecules involved in the development of atherosclerosis [5, 33]. In $\mathrm{ApoE}^{-1-}$ rats, impaired vascular compliance was associated with an increased inflammatory response, probably influencing aortic distensibility by inducing vascular remodeling processes. In the aorta of 16 weeks old preatherosclerotic $\mathrm{ApoE}^{-/-}$rats, IL1 $\beta$ was significantly 
up-regulated compared to SD rats, but transcription levels of TNF $\alpha$, and pro-atherosclerotic ICAM-1 and VCAM-1 were not significantly increased, underpinning the absence of atherosclerotic plaques in the aortic lumen. In isolated mononuclear cells (MNCs), mRNA expression of TNF $\alpha$ and IL6 was sensitive to RDN, and upregulation of these inflammatory marker genes could be inhibited. In contrast to MNCs, RDN did neither affect plasma levels of IL6 and IL1 $\beta$ nor gene expression of inflammatory cytokines in aortic tissue. Thus, in atherosclerosis prone $\mathrm{ApoE}^{-/-}$rats, $\mathrm{RDN}$ failed to improve overall inflammatory activation, triggered by hypercholesterolemia [34], but influenced activation of MNCs, while circulating interleukin levels and local aortic inflammatory activation was not modulated.

\section{Conclusions}

$\mathrm{ApoE}^{-1-}$ rats fed with standard chow at an age of 16 weeks develop impaired vascular compliance comparable to other ApoE-deficient animal models [2, 20], yet at an atherosclerosis prone stage. Sympathetic modulation by RDN prevented increasing aortic stiffening and positively effects endothelium-dependent relaxation in $\mathrm{ApoE}^{-1-}$, independently of alterations in BP and HR. These changes were associated with a reduction in aldosterone levels, and aortic fibrosis formation. Our data provide further evidence of the vital impact of the SNS in mediating vascular function and disease. The effects of SNS activation on vascular compliance are multifactorial. However, further experiments are needed to elucidate the involved pathways of the potential impact of RDN. At an age of 16 weeks hyperlipidemic $\mathrm{ApoE}^{-/-}$rats revealed decreased aortic distensibility, and impaired vasorelaxation, when fed with normal chow, thus providing a useful tool to study aortic compliance and vascular response at an atherosclerosis prone stage. Future studies are necessary to assess whether catheter-based RDN also exhibits beneficial effects on vascular compliance in patients with and without hypertension.

\section{Clinical perspective}

Several clinical trials have demonstrated that catheter based RDN reduces blood pressure in certain patients with resistant hypertension [9-12]. In contrast, the recently published SYMPLICITY 3 trial showed no significant difference in blood pressure between patients receiving sham treatment or RDN [35]. Reduction of afferent sympathetic nervous system activity and thereby central sympathetic activity may provide beneficial effects beyond lowering of blood pressure, including improvements in glucose tolerance [11], attenuation of the progression of atherosclerosis [18], reduction in left ventricular hypertrophy [36], and antiarrhythmic effects $[17,37]$. Studies in a rat model of metabolic syndrome, bilateral RDN significantly reduced plasma renin activity, and prevented progression of kidney injury and cardiac remodeling [16]. Early trials in surgical sympathetectomy in severe hypertension documented reduced morbidity and mortality even in patients without blood pressure changes following the operation [38]. We provide preclinical evidence suggestive of beneficial vascular effects associated with RDN, not exclusively related to alterations in blood pressure.

\section{Limitations}

At an age of 16 weeks hypercholesterolemic $\mathrm{ApoE}^{-/-}$rats demonstrated elevated plasma aldosterone levels compared to normocholesterolemic SD. This is in line with observations, that aldosterone production is sensitive to elevated low-density lipoprotein concentrations [39]. Herein, RDN did not affect plasma aldosterone concentrations in $\mathrm{SD}$, but prevented a progressive increase in $\mathrm{ApoE}^{-1-}$, preserving aldosterone levels comparable to $\mathrm{SD}$. The underlying mechanism remains speculative. In our normotensive rat model, renal tissue concentrations of NE, renin, and AngII did not differ between SD and $\mathrm{ApoE}^{-1-}$, and RDN caused a similar decrease of these parameters in both $\mathrm{SD}$ and $\mathrm{ApoE}^{-/-}$without significantly reducing blood pressure. This is in agreement with observations from Wang et al. [18], who documented significantly reduced concentrations in serum aldosterone, renal NE, and plasma renin in normotensive $\mathrm{ApoE}^{-1-}$ mice following RDN, however blood pressure and systemic concentrations of NE, renin, and AngII remained unaffected [18]. Whether the decrease in plasma aldosterone levels occurs indirectly due to systemic regulatory adaptive changes or via additional beneficial effects remains unclear and deserves further studies.

\section{Additional file}

Additional file 1. Complementary methods on aortic wall examination and oil-red O staining. Figure S1. Demonstrating the effect of $0.3 \%$ cholesterol on liver fat-content and on plaque formation in the thoracic aorta and aortic sinus. Figure S2. Depicts the examination of atherosclerotic plaques, elastic laminae and aortic wall thickness using either oil-red O staining, Hematoxylin and Eosin Staining or Elastica Van Gieson staining.

Table S1. Shows plasma concentration of inflammatory cytokines IL6 and IL $1 \mathrm{~b}$ and aortic gene expression of IL1b, TNFa, ICAM-1, VCAM-1 and eNOS.

\footnotetext{
Abbreviations

AF: atrial fibrillation; $\mathrm{ApoE}^{-/-}$: apolipoprotein E-deficient; BP: blood pressure; ED: end diastole; ES: end systole; eNOS: endothelial nitric oxide synthase; GAPDH: glyceraldehyd-3-phosphate-dehydrogenase; GPT: glutamat-pyruvattransaminase; GOT: glutamat-oxalacetat-transaminase; HDL: high-density lipoprotein; HPLC: high pressure liquid chromatography; HR: heart rate; ICAM-: intercellular adhesion molecule; IL $1 \beta$ : interleukin 1 beta; LDL: Iow-density lipoprotein; LVESV: left ventricular end-systolic volume; LVEDV: left ventricular
} 
end-diastolic volume; MNCs: mononuclear cells; MRI: magnetic resonance imaging; NE: norepinephrine; NO: nitric oxide; PP: pulse pressure; RAAS: renin-angiotensin-aldosterone-system; RDN: renal denervation; ROS: reactive oxygen species; SD: Sprague-Dawley; SNS: sympathetic nervous system; TNFa: tumor necrosis factor alpha; VCAM-1: vascular cell adhesion molecule-1.

\section{Authors' contributions}

MH, DL, DU and TS carried out molecular genetic studies, immunoassays, histology, performed the statistical analysis and drafted the manuscript. PF, AM and JS performed magnetic resonance imaging scans and analysed the data. $J \mathrm{G}$ and $\mathrm{BH}$ performed serum chemistry and norepinephrine content determination in rat kidney tissue. FM, MB, UL and SS made substantial contributions to conception and design and participated in the interpretation of the data and have been involved in critically revising the manuscript. All authors read and approved the final manuscript.

\section{Author details}

1 Klinik für Innere Medizin III, Universität des Saarlandes, 66421 Homburg/Saar, Germany. ${ }^{2}$ Klinik für Diagnostische und Interventionelle Radiologie, Universität des Saarlandes, Homburg/Saar, Germany. ${ }^{3}$ Klinik für Innere Medizin IV, Universität des Saarlandes, Homburg/Saar, Germany. ${ }^{4}$ Zentrallabor, Klinische Chemie und Laboratorium Medizin, Universität des Saarlandes, Homburg/Saar, Germany. ${ }^{5}$ Institut für Klinische Hämostaseologie und Transfusionsmedizin, Universität des Saarlandes, Homburg/Saar, Germany.

\section{Acknowledgements}

The authors thank Jeannette Zimolong, Kathrin Gaspard, Simone Jäger, Ellen Becker, Nina Rebmann, Sarah Triem and Christian Heugel for excellent technical assistance.

\section{Availability of data and supporting materials}

The dataset supporting the conclusions of this article is included within the article and in the Additional file 1.

\section{Competing interests}

The authors declare that they have no competing interests.

\section{Fundings}

This research was supported by the Deutsche Gesellschaft für KardiologieHerz und Kreislaufforschung.

\section{Ethical approval and consent to participate}

All animal experiments were conducted in accordance with "The guide for the Care and Use of laboratory Animals" published by the US National Institutes of Health (NIH Publication No. 85-23, revised 1996) and approved by the local animal ethics committee (Landesamt für Verbraucherschutz, Amtstierärztlicher Dienst, Lebensmittelüberwachung Zentralstelle, Saarbrücken, Germany. Nr:: 38/2012).

Received: 4 February 2016 Accepted: 18 May 2016

Published online: 08 June 2016

\section{References}

1. Cavalcante JL, Lima JA, Redheuil A, Al-Mallah MH. Aortic stiffness: current understanding and future directions. J Am Coll Cardiol. 2011. doi:10.1016/j.jacc.2010.12.017.

2. Wang $Y X$, Halks-Miller M, Vergona R, Sullivan ME, Fitch $R$, Mallari $C$, Martin-McNulty B, da Cunha V, Freay A, Rubanyi GM, Kauser K. Increased aortic stiffness assessed by pulse wave velocity in apolipoprotein E-deficient mice. Am J Physiol Heart Circ Physiol. 2000. doi:10.1161/ HYPERTENSIONAHA.114.04648.

3. Levenson J, Del Pino M, Razavian M, Merli I, Filitti V, Simon A. Hypercholesterolaemia alters arterial and blood factors related to atherosclerosis in hypertension. Atherosclerosis. 1992;95:171-9.

4. Miller FJ Jr, Gutterman DD, Rios CD, Heistad DD, Davidson BL. Superoxide production in vascular smooth muscle contributes to oxidative stress and impaired relaxation in atherosclerosis. Circ Res. 1998. doi:10.1161/01. RES.82.12.1298.
5. Zieman SJ, Melenovsky V, Kass DA. Mechanisms, pathophysiology, and therapy of arterial stiffness. Arterioscler Thromb Vasc Biol. 2005. doi:10.1161/01.ATV.0000160548.78317.29.

6. Wassmann S, Stumpf M, Strehlow K, Schmid A, Schieffer B, Böhm M, Nickenig $\mathrm{G}$. Interleukin-6 induces oxidative stress and endothelial dysfunction by overexpression of the angiotensin II type 1 receptor. Circ Res. 2004 doi:10.1161/01.RES.0000115557.25127.8D.

7. Dinenno FA, Jones PP, Seals DR, Tanaka H. Age-associated arterial wall thickening is related to elevations in sympathetic activity in healthy humans. Am J Physiol Heart Circ Physiol. 2000;278:1205-10.

8. Pauletto P, Scannapieco G, Pessina AC. Sympathetic drive and vascular damage in hypertension and atherosclerosis. Hypertension. 1991;17(Suppl 4):II175-81.

9. Azizi M, Sapoval M, Gosse P, Monge M, Bobrie G, Delsart P, Midulla M, Mounier-Véhier C, Courand PY, Lantelme P, Denolle T, DourmapCollas C, Trillaud H, Pereira H, Plouin PF, Chatellier G. Renal denervation for hypertension (DENERHTN) investigators. Optimum and stepped care standardised antihypertensive treatment with or without renal denervation for resistant hypertension (DENERHTN): a multicentre, open-label, randomised controlled trial. Lancet. 2015. doi:10.1016/ S0140-6736(14)61942-5.

10. Krum H, Schlaich MP, Sobotka PA, Böhm M, Mahfoud F, Rocha-Singh K, Katholi R, Esler MD. Percutaneous renal denervation in patients with treatment-resistant hypertension: final 3-year report of the symplicity HTN-1 study. Lancet. 2014. doi:10.1016/S0140-6736(13)62192-3.

11. Mahfoud F, Schlaich M, Kindermann I, Ukena C, Cremers B, Brandt MC, Hoppe UC, Vonend O, Rump LC, Sobotka PA, Krum H, Esler M, Böhm M. Effect of renal sympathetic denervation on glucose metabolism in patients with resistant hypertension: a pilot study. Circulation. 2011. doi:10.1161/CIRCULATIONAHA.110.991869.

12. Kopp UC, Jones SY, DiBona GF. Afferent renal denervation impairs baroreflex control of efferent renal sympathetic nerve activity. Am J Physiol Regul Integr Comp Physiol. 2008. doi:10.1152/ajpregu.90529.2008.

13. Böhm M, Linz D, Urban D, Mahfoud F, Ukena C. Renal sympathetic denervation: applications in hypertension and beyond. Nat Rev Cardiol. 2013. doi:10.1038/nrcardio.2013.89.

14. Linz D, Ukena C, Mahfoud F, Neuberger HR, Böhm M. Atrial autonomic innervation: a target for interventional antiarrhythmic therapy? J Am Coll Cardiol. 2014. doi:10.1016/j.jacc.2013.09.020.

15. Brandt MC, Reda S, Mahfoud F, Lenski M, Böhm M, Hoppe UC. Effects of renal sympathetic denervation on arterial stiffness and central hemodynamics in patients with resistant hypertension. J Am Coll Cardiol. 2012. doi:10.1016/j.jacc.2012.08.959.

16. Linz D, Hohl M, Schütze J, Mahfoud F, Speer T, Linz B, Hübschle T, Juretschke HP, Dechend R, Geisel J, Rütten H, Böhm M. Progression of kidney injury and cardiac remodeling in obese spontaneously hypertensive rats: the role of renal sympathetic innervation. Am J Hypertens. 2015. doi:10.1093/ajh/hpu123.

17. Linz D, van Hunnik A, Hohl M, Mahfoud F, Wolf M, Neuberger HR, Casadei B, Reilly SN, Verheule S, Böhm M, Schotten U. Catheter-based renal denervation reduces atrial nerve sprouting and complexity of atrial fibrillation in goats. Circ Arrhythm Electrophysiol. 2015. doi:10.1161/ CIRCEP.114.002453.

18. Wang H, Wang J, Guo C, Luo W, Kleiman K, Eitzman DT. Renal denervation attenuates progression of atherosclerosis in apolipoprotein E-deficient mice independent of blood pressure lowering. Hypertension. 2015. doi:10.1161/HYPERTENSIONAHA.114.04648.

19. Tomofuji T, Ekuni D, Azuma T, Irie K, Endo Y, Kasuyama K, Yoneda T, Morita M. Involvement of toll-like receptor 2 and 4 in association between dyslipidemia and osteoclast differentiation in apolipoprotein E deficient rat periodontium. Lipids Health Dis. 2013. doi:10.1186/1476-511X-12-1.

20. Schlimmer N, Kratz M, Böhm M, Baumhäkel M. Telmisartan, ramipril and their combination improve endothelial function in different tissues in a murine model of cholesterol-induced atherosclerosis. Br J Pharmacol. 2011. doi:10.1111/j.1476-5381.2011.01267.

21. Kannel WB. Office assessment of coronary candidates and risk factor insights from the Framingham study. J Hypertension. 1991;9:13-9.

22. Schlaich MP, Lambert E, Kaye DM, Krozowski Z, Campbell DJ, Lambert G, Hastings J, Aggarwal A, Esler MD. Sympathetic augmentation in hypertension: role of nerve firing, norepinephrine reuptake, and 
Angiotensin neuromodulation. Hypertension. 2004. doi:10.1161/01. HYP.0000103160.35395.9E.

23. Atlas SA. The renin-angiotensin aldosterone system: pathophysiological role and pharmacologic inhibition. J Manag Care Pharm. 2007;13(Suppl 8B):9-20.

24. Imanishi M, Tomita S, Ishizawa K, Kihira Y, Ueno M, Izawa-Ishizawa Y, Ikeda Y, Yamano N, Tsuchiya K, Tamaki T. Smooth muscle cell-specific Hif-1a deficiency suppresses angiotensin II-induced vascular remodelling in mice. Cardiovasc Res. 2014. doi:10.1093/cvr/cvu061.

25. Lin CX, Rhaleb NE, Yang XP, Liao TD, D'Ambrosio MA, Carretero OA. Prevention of aortic fibrosis by $\mathrm{N}$-acetyl-seryl-aspartyl-lysyl-proline in angiotensin II-induced hypertension. Am J Physiol Heart Circ Physiol. 2008. doi:10.1152/ajpheart.00481.2008.

26. Takai S, Jin D, Muramatsu M, Kirimura K, Sakonjo H, Miyazaki M. Eplerenone inhibits atherosclerosis in nonhuman primates. Hypertension. 2005. doi:10.1161/01.HYP.0000184640.81730.22.

27. Keidar S, Hayek T, Kaplan M, Pavlotzky E, Hamoud S, Coleman R, Aviram M. Effect of eplerenone, a selective aldosterone blocker, on blood pressure, serum and macrophage oxidative stress, and atherosclerosis in apolipoprotein E-deficient mice. J Cardiovasc Pharmacol. 2003:41:955-63.

28. Fitch RM, Rutledge JC, Wang YX, Powers AF, Tseng JL, Clary T, Rubanyi GM. Synergistic effect of angiotensin II and nitric oxide synthase inhibitor in increasing aortic stiffness in mice. Am J Physiol Heart Circ Physiol. 2006. doi:10.1152/ajpheart.00327.2005.

29. Harrison DG. Cellular and molecular mechanisms of endothelial cell dysfunction. J Clin Invest. 1997. doi:10.1172/JCI119751.

30. Wilkinson IB, Qasem A, McEniery CM, Webb DJ, Avolio AP, Cockcroft JR. Nitric oxide regulates local arterial distensibility in vivo. Circulation. 2002. doi:10.1161/hc0202.101970.

31. Mollnau H, Wendt M, Szöcs K, Lassègue B, Schulz E, Oelze M, Li H, Bodenschatz M, August M, Kleschyov AL, Tsilimingas N, Walter U, Förstermann U, Meinertz T, Griendling K, Münzel T. Effects of angiotensin II infusion on the expression and function of $\mathrm{NAD}(\mathrm{P}) \mathrm{H}$ oxidase and components of nitric oxide/cGMP signaling. Circ Res. 2002;90:E58-65. doi:10.1161/01. RES.0000012569.55432.02.
32. Libby P. Inflammation in atherosclerosis. Nature. 2002. doi:10.1038/ nature01323.

33. Viel EC, Lemarié CA, Benkirane K, Paradis P, Schiffrin EL. Immune regulation and vascular inflammation in genetic hypertension. Am J Physiol Heart Circ Physiol. 2010. doi:10.1152/ajpheart.00707.2009.

34. Steinberg D. Hypercholesterolemia and inflammation in atherogenesis: two sides of the same coin. Mol Nutr Food Res. 2005. doi:10.1002/ mnfr.200500081.

35. Bhatt DL, Kandzari DE, O'Neill WW, D'Agostino R, Flack JM, Katzen BT, Leon MB, Liu M, Mauri L, Negoita M, Cohen SA, Oparil S, Rocha-Singh K, Townsend RR, Bakris GL. SYMPLICITY HTN-3 investigators. A controlled trial of renal denervation for resistant hypertension. N Engl J Med. 2014 doi:10.1056/NEJMoa1402670.

36. Mahfoud F, Urban D, Teller D, Linz D, Stawowy P, Hassel JH, Fries P, Dreysse S, Wellnhofer E, Schneider G, Buecker A, Schneeweis C, Doltra A, Schlaich MP, Esler MD, Fleck E, Böhm M, Kelle S. Effect of renal denervation on left ventricular mass and function in patients with resistant hypertension: data from a multi-centre cardiovascular magnetic resonance imaging trial. Eur Heart J. 2014. doi:10.1093/eurheartj/ehu093.

37. Linz D, Hohl M, Nickel A, Mahfoud F, Wagner M, Ewen S, Schotten U, Maack C, Wirth K, Böhm M. Effect of renal denervation on neurohumoral activation triggering atrial fibrillation in obstructive sleep apnea. Hypertension. 2013. doi:10.1161/HYPERTENSIONAHA.113.01728.

38. Smithwick RH, Thompson JE. Splanchnicectomy for essential hypertension. JAMA. 1953;152:1501-4.

39. Nishikawa T, Suematsu S, Saito J, Soyama A, Ito H, Kino T, Chrousos G. Human renal mesangial cells produce aldosterone in response to lowdensity lipoprotein (LDL). J Steroid Biochem Mol Biol. 2005;96:309-16.

\section{Submit your next manuscript to BioMed Central and we will help you at every step:}

- We accept pre-submission inquiries

- Our selector tool helps you to find the most relevant journal

- We provide round the clock customer support

- Convenient online submission

- Thorough peer review

- Inclusion in PubMed and all major indexing services

- Maximum visibility for your research

Submit your manuscript at www.biomedcentral.com/submit
() Biomed Central 